\title{
Inotersen Treatment for Patients with Hereditary Transthyretin Amyloidosis
}

\author{
Herediter Transtiretin Amiloidozis Hastalarında İnotersen Tedavisinin \\ Etkinliği ve Güvenilirliği
}

\author{
(1) Faruk Uğur Doğan, (1) Murat Kürtüncü \\ Istanbul University Istanbul Faculty of Medicine, Department of Neurology, Istanbul, Turkey
}

Keywords: Inotersen, hereditary transthyretin amyloidosis, polyneuropathy

Anahtar Kelimeler: İnotersen, herediter transtiretin amiloidozis, polinöropati

\section{Inotersen Treatment for Patients with Hereditary Transthyretin Amyloidosis}

Hereditary transthyretin (TTR) amyloidosis (HTA) is characterized by the accumulation of the incorrectly folded protein as amyloid deposits in extracellular space as a result of the mutation of the gene coding transthyretin (TTR) (1). The accumulation of amyloid deposits in various organs causes peripheral neuropathy, cardiomyopathy, nephropathy and gastrointestinal dysfunction (2). Inotersen is an antisense oligonucleotide molecule that reduces the production of TTR in the liver (3).

Ackermann et al. (3) investigated the efficacy and reliability of inotersen on the polyneuropathy caused by HTA in the study NEURO-TTR, which was published in the New England Journal of Medicine. Twenty-four centers from 10 countries between March 2013 and April 2017 were included in the study. Patients with TTR mutation and polyneuropathy due to HTA who could walk without aid (stage 1) and who could walk with aid (stage 2) aged 18-82 years were included in the study. Patients with other diseases that might cause polyneuropathy, with liver transplantation, and patients having stage 3 or higher disease according to the New York Heart Association classification were excluded. During the study, patients were not allowed to receive diflunisal or tafamidis as additional therapy.

In the study, patients received $300 \mathrm{mg}$ inotersen subcutaneously or placebo, 3 times in the first week and once per week in the following 64 weeks. In order to prevent vitamin A deficiency in tissue due to decreased levels of TTR, approximately $3000 \mathrm{IU}$ vitamin A per day was given to patients. Patients were divided into subgroups according to whether they had the Val30Met mutation, stage 1 or stage 2 disease, previous treatment, and cardiomyopathy.

The Modified Neuropathy Impairment Score +7 (mNIS +7$)$ and the Norfolk Quality of Life-Diabetic Neuropathy (QOL$\mathrm{DN}$ ) questionnaire were used as primary evaluation scales. It was accepted that lower scores on both scales showed better clinical status and quality of life.

One hundred seventy-two patients were included in the study. The patients were randomized into 2 groups: placebo $(n=60)$ and inotersen $(n=112)$. One hundred thirty-nine patients were able to complete the study. The most important reason for discontinuing with the study was adverse effects of the drug in the inotersen group $(n=16)$ and disease progression $(n=3)$ in the placebo group.

At the end of 66 weeks, the change in mNIS +7 score in the inotersen group compared with the placebo group was found as 7.7 points lower, and the change in the Norfolk QOL-DN scale was 11.7 points lower. As a result, it was shown that inotersen significantly reduced the progression of the disease. In some of the patients who completed the study in the inotersen group (36\% of the patients in the $\mathrm{mNIS}+7$ scale and $50 \%$ in the Norfolk QoL-DN questionnaire), the score at the beginning of treatment did not change. Inotersen was shown to significantly slow down the progression of the disease in all previously defined sub-groups. However, there were no differences between the two groups in terms of the global longitudinal strain and other echocardiographic parameters. It was found that inotersen had a positive effect on cachexia, which is an important cause of mortality in HTA by slowing down weight loss.

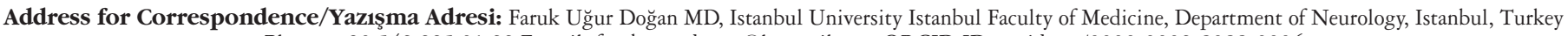
Phone: +905422950122 E-mail: farukugurdogan@hotmail.com ORCID ID: orcid.org/0000-0002-8088-0006

Received/Geliş Tarihi: 25.12.2018 Accepted/Kabul Tarihi: 07.01.2019

${ }^{\circ}$ Copyright 2019 by Turkish Neurological Society

Turkish Journal of Neurology published by Galenos Publishing House. 
It was observed that the serum TTR level fell to $79 \%$ of the baseline value at the $13^{\text {th }}$ week and stayed stable until the $65^{\text {th }}$ week, and that there was no correlation between the change in serum TTR level and the changes in the scales used.

There were five deaths during the study period. All deaths were in the inotersen group, one of which was due to fatal intracranial hemorrhage due to severe thrombocytopenia $\left(<10,000 / \mathrm{mm}^{3}\right)$. The other four were associated with the progression of the disease. Improvement in thrombocytopenia developed in patients with glucocorticoid therapy and the existence of antiplatelet immunoglobulin $G$ suggested that thrombocytopenia could develop due to immune mechanisms. Glomerulonephritis was detected in three patients; kidney functions were restored in two patients with immunosuppressive therapy, and in one patient the need for permanent hemodialysis was born.

As a result, this study demonstrated the benefit of weekly subcutaneous inotersen treatment in HTA polyneuropathy both in clinical evaluation and in quality of life scales. Early detection of thrombocytopenia and glomerulonephritis through close followup can also make the treatment of these complications possible. Exclusion of patients with severe disease and lack of parameters showing the efficacy of inotersen on cardiomyopathy were the limitations of the study.

\section{References}

1. Sekijima Y. Transthyretin (ATTR) amyloidosis: clinical spectrum, molecular pathogenesis and disease-modifying treatments. J Neurol Neurosurg Psychiatry 2015;86:1036-1043.

2. Plante-Bordeneuve V, Said G. Familial amyloid polyneuropathy. Lancet Neurol 2011;10:1086-1097.

3. Ackermann EJ, Guo S, Benson MD, et al. Suppressing transthyretin production in mice, monkeys and humans using 2 nd Generation antisense oligonucleotides. Amyloid 2016;23:148-157. 